\title{
Fuzzy PID Control Simulation Research on Rotational Speed Regulation for Diesel Engines
}

\author{
Leyu ZHANG \\ Department of Electric Power Engineering \\ Chongqing Communication Institute \\ Chongqing, China \\ Leyuzhang2014@163.com \\ Lin XIAO \\ Department of Electric Power Engineering \\ Chongqing Communication Institute \\ Chongqing, China \\ Xiaolin_cci@163.com
}

\author{
Zhao JIN \\ Department of Electric Power Engineering \\ Chongqing Communication Institute \\ Chongqing, China \\ ZJIN2010@163.com
}

\begin{abstract}
The online self-adjustment of parameters of diesel engines mechanical speed controller is achieved by a designed fuzzy PID controller, through establishing a mathematic model of diesel engine mechanical centrifugal speed controller and the application of the advantages of the conventional PID control and fuzzy control. The fuzzy PID diesel engine mechanical speed control system is simulated by Simulink of MATLAB. The digital electronic governor depends on controlling software to realize the function of regulation and control. It can automatically regulate the accelerograph of the diesel engine, gives the engine the right amount fuel oil, and lets the diesel engine work at the fixed rotational speed when the load is being changed. It also has solved the mechanical governor problems such as poor accuracy and large time consumption to achieve stable state, and it is versatile and possesses extensional ability. The simulation results suggest fine dynamic performance, strong robustness, and better adaptability, compared to the conventional PID control algorithm of fuzzy self-adjustment PID algorithm. The performance of diesel engine speed control system is thusly largely advanced.
\end{abstract}

Keywords-Diesel Engine; Rotational Speed Regulation; Fuzzy PID Control Algorithm; Mathematic Model; Simulation of Control System

\section{INTRODUCTION}

In order to obtain stable rotation speed of diesel engine under various loadings speed controllers are indispensable. The typically used speed controllers are mechanical centrifugal speed controller which could control the openness of the fuel injection pump by the centrifugal forces contributed by fly balls ${ }^{1-2]}$. Adopting the conventional single PID control method is hard to satisfy the requirement of diesel engine speed control because the system is complex a nonlinear multi-lever type. Thus, a new method, by combining the fuzzy control and conventional PID control in order to accomplish the online adjustment of parameters of PID controller, is established. The advancement of the new fuzzy PID controller is well testified by the performance comparison the conventional PID control and the new method ${ }^{[3-5]}$.

Diesel engine generator is an equipment that produces exportable electricity, it works by diesel engine driving a dynamo to work. The dynamo is normally a synchronous AC generator, the output frequency is related to rotational speed of diesel engine. To guarantee the power quality of generator, a speed controller is needed to adjust the rotation speed of Rotational engine. At present, most of the speed controller is mechanical governor, because the mechanical governor itself has the defect of hysteretic inertia and big frictional resistance, it inevitably has the weakness of long adjust time, poor stability and poor reliability ${ }^{[6]}$. With the fast development of economic and society, the use of combustion engines has become increasingly prevalent, the energy and environmental problems become more and more prominent, the higher requirement about the control accuracy and discharge of combustion engine is expected. The digital electronic governor is the advanced governing system, can vastly improve the speed adjusting performance of diesel engine generator, makes up the inherent deficiency of mechanical governor, and the governor can be improved by using advanced control method. At present, the similar digital electronic governors on the market are all used for diesel, can' t directly use for diesel engine. So it is imperative to develop a new digital electronic governor with high reliability, high generality and reasonable price, and it will have broad market prospect ${ }^{[7-9]}$.

\section{MATHEMATICAL MODEL OF DIESEL ENGINES}

When establishing diesel engine mechanical centrifugal speed controller, a comprehensive consideration regarding draw bar displacements of per circulation fuel injection pump of diesel engine, oil supply variation caused by the rotation variation, and the coming energy transmission, is needed to the noted. In the mathematic model the diesel engine and loading bearing equipment are considered. The diesel speed control system is established by loading the 
sudden change loading as the disturbing signals. See the detail in Fig. 1 .

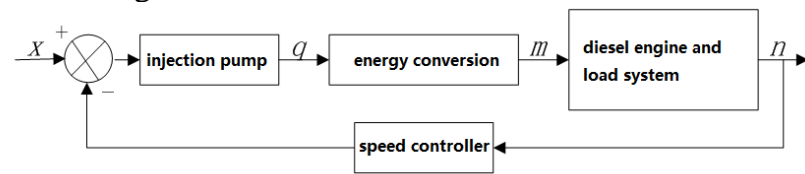

Figure 1. Diesel Engine Speed Control System

The analysis of speed control system is facilitated by establishing mathematical model. The equation of fuel injection pump draw bar displacement variation and rotation variation of diesel engine rotation speed deviation is $[10,11]$.

$$
m \frac{d^{2} x(t)}{d t^{2}}+c \frac{d x(t)}{d t}+\left(k-a \frac{\partial F}{\partial R}\right) x(t)=r \frac{\partial F}{\partial n} n(t)
$$

Where: $\mathrm{r}$ - ratio of injection pump draw bar displacement $x$ and sliding sleeve displacement $z$, here the number is $r=2 ; n-$ diesel generator rotation speed, the value here is $1500 \mathrm{r} / \mathrm{min} ; n(t)$ - diesel generator output rotation speed deviation given rotation error variation, $\mathrm{r} / \mathrm{min} ; m$ equivalent mass of the motion components translated into sliding sleeve shaft, $\mathrm{kg}$, the value is $m=1.52 \mathrm{~kg}$, based on equivalent weight kinetic energy; $k$-equivalent weight stiffness of springs of the motion components translated into sliding sleeve shaft, $k=5368 \mathrm{~N} / \mathrm{m} ; R$ - Radius of gyration center of mass of fly balls, the value can be calculated by structural parameters, i.e. $\mathrm{R}=0.0238 \mathrm{~m} ; a$ Ration of radius of gyration center of mass of fly balls and sliding sleeve displacement, $a=0.785 ; F$ - centrifugal inertia force of fly ball, $F=m_{l} R w^{2}, \mathrm{~m}_{1}$ is the total mass of fly balls, $m_{l}=0.73 \mathrm{~kg}, w$ is rate of angular motion of governor shaft, $\mathrm{rad} / \mathrm{s} ; c-$ The equivalent viscous damping coefficient of governor Reciprocating motion components translated into sliding sleeve shaft, $\mathrm{N} \cdot \mathrm{s} / \mathrm{m}$; the value her is $\mathrm{c}=450 \mathrm{~N} \cdot \mathrm{s} / \mathrm{m}$.

Fuel-injection quantity variation and draw bar displacement variation and diesel engine rotation speed deviation given rotation speed variation equation of motion is:

$$
q(t)=\frac{\partial Q}{\partial x} x(t)+\frac{\partial Q}{\partial n} n(t)=k_{1} x(t)+k_{2} n(t)
$$

Where, $q(t)$-Fuel-injection quantity variation per working circle of the diesel generator, $\mathrm{kg} / \mathrm{cyc} ; Q-$ Fuelinjection quantity for the diesel generator per circle in stable condition, $\mathrm{kg} / \mathrm{cyc} ; k_{l}$ - rate of change of fuelinjection quantity to draw bar displacement, mainly related to plunger chute angle. The value is $k_{1}=9.43 \times 10^{-8} \mathrm{~kg} /(\mathrm{cyc} \cdot \mathrm{m}) ; k_{2}$-rate of change of fuel-injection quantity to generator rotation speed, mainly related to oil pump property. The value is $k_{2}=7.23 \times 10^{-9} \mathrm{~kg} \cdot \mathrm{min} / \mathrm{cyc}$.

The signaling torsion variation of the diesel generator and fuel-injection quantity equation of motion is:

$$
M_{i}(t)=\frac{\partial M}{\partial Q} q(t)=k_{3} q(t)
$$

Where, $\mathrm{M}$ - signaling torsion of the diesel generator $\mathrm{N} \cdot \mathrm{m} ; M_{i}(\mathrm{t})$ - signaling torsion variation of the diesel generator, $\mathrm{N} \cdot \mathrm{m} / \mathrm{cyc} ; k_{3}$ - equivalent coefficient, $k_{3}=\frac{\partial M}{\partial Q}=3.58 \times 10^{6} \mathrm{~N} \cdot \mathrm{m} / \mathrm{kg}$

The diesel generator rotation speed deviation given rotation speed variation and signaling torsion variation of the diesel generator equation of motion is:

$$
M_{i}(t)=\frac{\pi J}{30} \bullet \frac{d n(t)}{d t}+\frac{\partial M_{m}}{\partial n} n(t)=\frac{\pi J}{30} \bullet \frac{d n(t)}{d t}+k_{4} n(t)
$$

Where, $J$ - moment of inertia of the diesel generator set, $\mathrm{kg} \cdot \mathrm{m}^{2}$, the value is $\mathrm{J}=1.43 \mathrm{~kg} \cdot \mathrm{m}^{2}$ when the rotation speed is $1500 \mathrm{r} / \mathrm{min} ; M_{m}$ - diesel generator friction torsion moment, the value is $\mathrm{M}_{\mathrm{m}}=36.2 \mathrm{~N} \cdot \mathrm{mwhen}$ the rotation speed is $1500 \mathrm{r} / \mathrm{min} ; k_{4}$ - damping coefficient of the diesel generator.

For equation (1) to equation (4), the transmission functions are obtained based on Laplace Transformation. The transmission function figure of the diesel engine speed control system is obtained by assuming $A=r \frac{\partial F}{\partial n}, E=a \frac{\partial F}{\partial R}$.

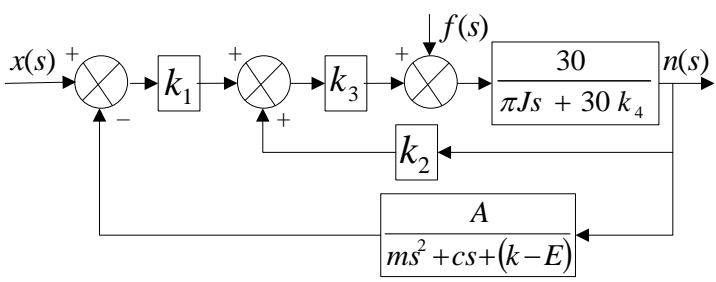

Figure 2. Transmission Function Figure of the Diesel Engine Speed Control System

In Fig .2, $f(s)$ is disturbing signal of mutational loadings and the system open-loop transfer function without disturbing signal is:

$$
G_{k}(s)=G(s) H(s)=\frac{30 A k_{1} k_{3}}{\left(m s^{2}+c s+k-E\right)\left(\pi J s+30 k_{4}-30 k_{2} k_{3}\right)}
$$

And the system close-loop transfer function is:

$$
\frac{n(s)}{x(s)}=\frac{30 A k_{1} k_{3}\left(m s^{2}+c s+k-E\right)}{30 A k_{1} k_{3}+\left(m s^{2}+c s+k-E\right)\left(\pi J s+30 k_{4}-30 k_{2} k_{3}\right)}
$$

It can be seen from the diesel speed control system transfer functions that the system is a complex nonlinear one and thus the conventional control system is hard to satisfy the dynamic characteristics. So an advanced intelligent control algorithm is needed.

\section{DESIGN OF FUZZY PID CONTROLLER}

\section{A. System Structure of Fuzzy PID Control}

A fuzzy PID control system which is to control the diesel engine mechanical centrifugal rotation speed is used in the paper. The fuzzy control, based on fuzzy sets theory, fuzzy language variation, and fuzzy logical reasoning, is a relatively mathematical method that can directly express the human empirical reasoning, judicial thinking process. Its control performance is comparatively well because the establishment of the control system is resulted from control principle by the language variable conditional statements ${ }^{[12-14]}$. 
The diesel rotation speed error $e$ and error change rate $e c$ of the processing system will be collected continuously for diesel mechanical centrifugal speed control self-adjustment control process. The modified parameters will be obtained on the condition that the system has experienced of fuzzificaiton, fuzzy reasoning, and defuzzificaiton by the fuzzy controller. The feedback of control signal to realize close loop control will be achieved by the final online self-adjustment of PID controller. The corresponding fuzzy PID control process is shown in Fig .3.

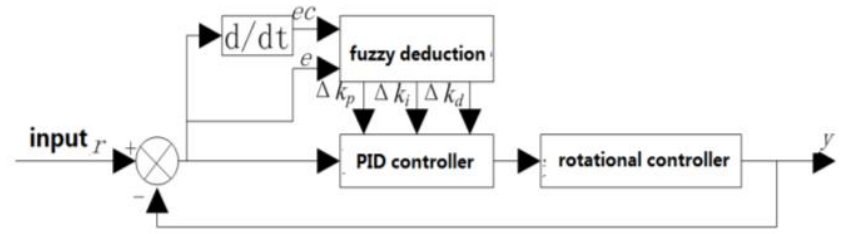

Figure 3. Fuzzy PID control process of diesel engine mechanical centrifugal controller

B. Membership Functions of Each Variable Quantities

\begin{tabular}{|c|c|c|c|c|c|c|}
\hline $\begin{array}{l}\mathrm{EC} \\
\mathrm{E}\end{array}$ & NB & NM & NS & $\mathrm{ZO}$ & PS & PM \\
\hline$\overline{\mathrm{NB}}$ & $\mathrm{PB} / \mathrm{NB} / \mathrm{PS}$ & $\mathrm{PB} / \mathrm{NB} / \mathrm{NS}$ & $\mathrm{PM} / \mathrm{NM} / \mathrm{NB}$ & $\mathrm{PM} / \mathrm{NM} / \mathrm{NB}$ & $\mathrm{PS} / \mathrm{NS} / \mathrm{NB}$ & $\mathrm{ZO} / \mathrm{ZO} / \mathrm{NM}$ \\
\hline NM & $\mathrm{PB} / \mathrm{NB} / \mathrm{PS}$ & $\mathrm{PB} / \mathrm{NB} / \mathrm{NS}$ & $\mathrm{PM} / \mathrm{NM} / \mathrm{NB}$ & PS/NS/NM & $\mathrm{PS} / \mathrm{NS} / \mathrm{NM}$ & $\mathrm{ZO} / \mathrm{ZO} / \mathrm{NS}$ \\
\hline NS & $\mathrm{PM} / \mathrm{NB} / \mathrm{ZO}$ & $\mathrm{PM} / \mathrm{NM} / \mathrm{NS}$ & $\mathrm{PM} / \mathrm{NS} / \mathrm{NM}$ & PS/NS/NM & $\mathrm{ZO} / \mathrm{ZO} / \mathrm{NS}$ & NS/PS/NS \\
\hline $\mathrm{ZO}$ & $\mathrm{PM} / \mathrm{NM} / \mathrm{ZO}$ & $\mathrm{PM} / \mathrm{NM} / \mathrm{NS}$ & $\mathrm{PS} / \mathrm{NS} / \mathrm{NS}$ & $\mathrm{ZO} / \mathrm{ZO} / \mathrm{NS}$ & NS/PS/NS & $\mathrm{NM} / \mathrm{PM} / \mathrm{NS}$ \\
\hline PS & $\mathrm{PS} / \mathrm{NM} / \mathrm{ZO}$ & $\mathrm{PS} / \mathrm{NS} / \mathrm{ZO}$ & $\mathrm{ZO} / \mathrm{ZO} / \mathrm{ZO}$ & $\begin{array}{l}\text { NS/PS/ZO } \\
\text { NST }\end{array}$ & NS/PS/ZO & NM/PM/ZO \\
\hline PM & $\mathrm{PS} / \mathrm{ZO} / \mathrm{PB}$ & $\mathrm{ZO} / \mathrm{ZO} / \mathrm{PS}$ & NS/PS/PS & NM/PS/PS & $\mathrm{NM} / \mathrm{PM} / \mathrm{ZO}$ & NM/PB/PS \\
\hline $\mathrm{PB}$ & $\mathrm{ZO} / \mathrm{ZO} / \mathrm{PB}$ & $\mathrm{ZO} / \mathrm{ZO} / \mathrm{PM}$ & NM/PS/PM & $\mathrm{NM} / \mathrm{PM} / \mathrm{PM}$ & $\mathrm{NM} / \mathrm{PM} / \mathrm{PS}$ & $\mathrm{NB} / \mathrm{PB} / \mathrm{PS}$ \\
\hline
\end{tabular}

Double-input treble-output structure is applied for diesel mechanical centrifugal speed control selfadjustment PID control process. The rotation speed error $e$ and error change rate $e c$ are fuzzy input variables while the three modified values of control parameters $\Delta k_{p}, \Delta k_{i}$, and $\Delta k_{d}$ are fuzzy output variables and their corresponding language variables are $\mathrm{E}, \mathrm{EC}, \Delta K_{P}, \Delta K_{I}$, and $\Delta K_{D}$. The fuzzy subset of diesel engine speed control system input and output variable language values are: $\{$ positive big(PB), positive middle(PM), positive small(PS), zero(ZO), negative small(NS), negative middle(NM), negative $\operatorname{big}(\mathrm{NB})\}$ while input and output variables fuzzy set discrete domain is: $\{-6,-4,-2,0,2,4,6\}$. The membership function is taken from Gaussian Function and the established membership function compile interface created in MATLAB software is shown in Fig .4.

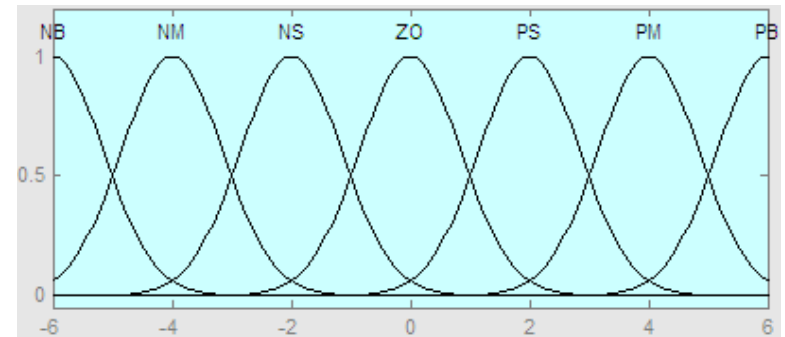

Figure 4. Membership function of Diesel Mechanical Centrifugal Speed Controller established in MATLAB

\section{Fuzzy Control Principle Table}

The establishment of fuzzy control principle table is mainly based on the practical conducting experience and the PID adjustment principle. See the fuzzy principle table of diesel mechanical centrifugal speed control in Tab.1.

TABEL 1 Fuzzy Control Principle Table for $\Delta \mathrm{KP}, \Delta \mathrm{KI}$ and $\Delta \mathrm{KD}$
As a bridge of linking continuous input variables and output variables, the fuzzy control principle is usually expressed as a sentence whose type is 'if A and B then C'. The fuzzy control principle could thusly be obtained on the basis of fuzzy control principle table. There are 49 fuzzy control principles since both the diesel rotation speed error $e$ and error change rate $e c$ include seven language variable quantities. Thus:

1. If ( $\mathrm{E}$ is $\mathrm{NB})$ and $(\mathrm{EC}$ is $\mathrm{NB})$ then $(\mathrm{KP}$ is $\mathrm{PB})(\mathrm{KI}$ is $\mathrm{NB})(\mathrm{KD}$ is $\mathrm{PS})$

2. If ( $\mathrm{E}$ is $\mathrm{NB})$ and (EC is $\mathrm{NM})$ then $(\mathrm{KP}$ is $\mathrm{PB})(\mathrm{KI}$ is $\mathrm{NB})(\mathrm{KD}$ is NS)

3. If (E is $\mathrm{NB})$ and (EC is NS) then (KP is $\mathrm{PM})(\mathrm{KI}$ is $\mathrm{NM})(\mathrm{KD}$ is $\mathrm{NB})$

4. If ( $\mathrm{E}$ is $\mathrm{NB})$ and $(\mathrm{EC}$ is $\mathrm{ZO})$ then $(\mathrm{KP}$ is $\mathrm{PM})(\mathrm{KI}$ is $\mathrm{NM})(\mathrm{KD}$ is $\mathrm{NB})$

5. If (E is $\mathrm{NB})$ and (EC is $\mathrm{PS})$ then (KP is $\mathrm{PS})(\mathrm{KI}$ is $\mathrm{NS})(\mathrm{KD}$ is NB)

6. If ( $\mathrm{E}$ is $\mathrm{NB})$ and (EC is $\mathrm{PM})$ then $(\mathrm{KP}$ is $\mathrm{ZO})(\mathrm{KI}$ is $\mathrm{Z})(\mathrm{KD}$ is $\mathrm{NM})$

7. If (E is $\mathrm{NB})$ and (EC is $\mathrm{PB})$ then $(\mathrm{KP}$ is $\mathrm{ZO})(\mathrm{KI}$ is $\mathrm{Z})(\mathrm{KD}$ is $\mathrm{PS})$

8. If ( $\mathrm{E}$ is $\mathrm{NM})$ and $(\mathrm{EC}$ is $\mathrm{NB})$ then $(\mathrm{KP}$ is $\mathrm{PB})(\mathrm{KI}$ is $\mathrm{NB})(\mathrm{KD}$ is PS)

......

49. If ( $\mathrm{E}$ is $\mathrm{PB})$ and $(\mathrm{EC}$ is $\mathrm{PB})$ then $(\mathrm{KP}$ is $\mathrm{NB})(\mathrm{KI}$ is $\mathrm{PB})(\mathrm{KD}$ is $\mathrm{PB})$ )

The fuzzy judgment needed to be solved in order to transform the fuzzy values into precise values after the fuzzy principle and fuzzy reasoning. The paper adopts the maximum membership method to conduct the fuzzy judgment on the basis of the practical conditions and the fuzzy control values of each parameter are obtained and the parameters are controlled through online checking ${ }^{[10]}$.

\section{Simulation RESEARCH OF DiESEL ENGINE ROTATIONAL SPEED CONTROL SYSTEM}

The simulation was conducted using Simulink, which is a data flow graphical programming language tool for modeling, simulating and analyzing, mainly for multidomain dynamic systems. It offers tight integration with the rest of the MATLAB environment and can either drive MATLAB or be scripted from it. Its primary interface is a graphical block diagramming tool and a customizable set of block libraries. Simulink is widely used in control theory and digital signal processing for multi-domain simulation and Model-Based Design.

Simulink Verification and Validation enables systematic verification and validation of models through modeling style checking, requirements trace ability and model coverage analysis. Simulink Design Verifier uses formal methods to identify design errors like integer overflow, division by zero and dead logic, and generates test case scenarios for model checking within the Simulink environment.

The simulation model of the fuzzy PID control and conventional PID control are established in Simulink on the basis of diesel engine speed control transfer function. Simulation curves of the two methods are shown in Fig .5. 


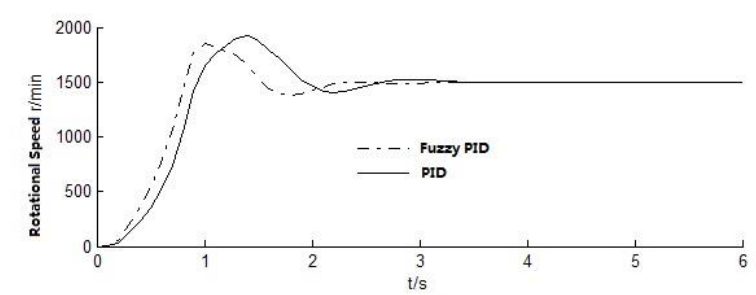

Figure 5. Diesel Rotation Curves by Fuzzy PID and Conventional PID Control Methods

It can be concluded from the above figure that the by the fuzzy PID method the system could response fast within one second and the output maximum overshoot are not more than $20 \%$. The system modifying is achieved within 2.5 seconds and then the system is witnessed no obvious fluctuation and reach stable state within a short period of time and the rotation speed of the diesel engine is stable at around $1500 \mathrm{r} / \mathrm{min}$ finally. As a contrast, if the conventional PID method is employed, the system accomplishes the response in 1.2 seconds and the output maximum overshoots are larger than those obtained in fuzzy PID control method and the control system needs 3.5 seconds for adjustment. After adjusting, there still exits some fluctuation and the rotation speed of the diesel generator reaching around $1500 \mathrm{r} / \mathrm{min}$ costs about 5 seconds. It is clear that the control effect of the fuzzy PID control method is much better than the conventional PID method.

\section{CONCLUSION}

In the paper a mathematical model for the diesel engine speed control system is established for checking the control effect of the fuzzy PID control method and the conventional PID method.

In the analyses of the gasoline characteristics, we obtained that the working process of gasoline has uncertainty, time invariance and nonlinearity, it is hard to establish the accurate mathematical model of gasoline engine, for the particular system the paper design a governor for gasoline generator based on fuzzy sliding mode control. PID model variable control has the advantage of fast response, good robustness, without online identification, and simple physical implementation. The only disadvantage is the chattering phenomenon. For the problem, the article introduce fuzzy control into sliding mode control, using fuzzy control rule to soften control signal, which can weaken the chattering phenomenon. Because the main disturbance in the system is load mutation, the paper design a feed forward compensation control system based on load mutation. Finally, the simulation of the designed controller is carried out in MATLAB.

According to the designed controller, the paper give the method and process of hardware design and software design. Hardware design mainly include: controller module, measure of rotate speed module, actuator module, led display module, power module, and current measurement module. Software design mainly include: control strategy calculation module, idling module, start module, halt module, load current measurement module and display module.
Installing the designed digital electronic governor on the selected diesel engine generator to carry out experiment. The result show that the digital electronic governor has better steady-state speed performance and dynamic speed governing performance. The governor has simple design, high reliability and big practical value.

The fuzzy PID diesel engine mechanical speed control system is simulated by Simulink and the simulation results suggest good dynamic performance, strong robustness, and better adaptability compared to the conventional PID control of fuzzy self-adjustment PID. The fuzzy PID control method can be used in the complex nonlinear diesel engine speed control system very well. When adopted in the diesel engine speed control system, the oscillation could be effectively reduced, the response speed could be well accelerated, and the stable accuracy could be increased and the dynamic property could be advanced. The method owns well control performance and can adapt to the system loading variation.

\section{References}

[1] [1] Chen Mao, Energy saving design of mechanical speed diesel generators[J]. Fujiang agricultural machinery, 2013, 4:46-48.

[2] [2] He Xinjun, diesel generator generator speed regulating system fuzzy self-adjustment PID control[D]. Chongqing university master's degree thesis, 2008

[3] Tian Fan. Electro hydraulic servo system fuzzy PID control simulation and testing research[D] University of Technology master's degree thesis, 2010 .

[4] Abdelhameed,M.M.Enhancement of sliding mode controller by fuzzy logic with application to robotic manipulators, Mechatrionics,2005,15:439-458.

[5] Abdelhameed,M.M. Enhancement of sliding mode controller by fuzzy logic with application to robotic manipulators, Mechatrionics,2005,15:439-458.

[6] Qiang LIgang, Li Xiangun. Continuous Casting mold nonsinusoidal oscillation system simulation research based on fuzzy self-adjustment PID control method[J]. Machine Tool \& Hydraulics, 2010,15:92-94

[7] Hua Yinan. Application of genetic optimized fuzzy PID control in diesel generator rotation speed control[J]. Electrometallurgy, 2013,32(16):26-29

[8] Woodward Governor Company, 723 digital speed control for reciprocating engines-DSLC ${ }^{\mathrm{TM}}$, Capatible Operation and Calibration Manual, 1996

[9] Dang Jianwu. Fuzzy control technology[M]. Beijing: China railway Publishing House, 2007.8

[10] Rojko,A.and Jezernik,K. Sliding-mode motion controller with adaptive fuzzy disturbance estimation,IEEE Transactions on Industrical Electronics,2004,51(5).

[11] Wu Xiaoli,v Lin Zhehui. MATLAB assistance fuzzy system design[M]. Xian: Xidian University press,2002

[12] Shtessel Y B, Zinober A S I, Shkolnikov I A. Sliding mode control for nonlinear systems with output delay via method of stable center[J]. Journal of Dynamic Systems, Measurement, and Control, 2003, 125: 158-165.

[13] Xia Y Q, Yingming Jia. Robust sliding-mode control for uncertain time-delay systems: a LMI approach[J]. Automatic Control, IEEE Transactions on, 2003, 48(6): 1086-1091.

[14] Ha,Q.P.,Rye,D.C.and Durrant-Whyte H.F. Fuzzy moving sliding control with application to robotic manipulators, Automatica,1999,35:607-616. 